\title{
ELECTRODINÁMICA QUIRAL: ESLABÓN PARA LA UNIFICACIÓN DEL ELECTROMAGNETISMO Y LA GRAVITACIÓN
}

\section{CHIRAL ELECTRODYNAMIC: CONNECTION FOR THE UNIFICATION OF ELECTROMAGNETISM AND GRAVITATION}

\author{
H. Torres-Silva ${ }^{1}$ \\ Recibido el 5 de septiembre de 2007, aceptado el 12 de diciembre de 2007 \\ Received: September 5, 2007 Accepted: December 12, 2007
}

\begin{abstract}
RESUMEN
Una alternativa a la teoría cuántica de la gravedad, aún no descubierta, es la Teoría Total Simplificada (TTS) aquí propuesta, que postula unificar la gravedad con el electromagnetismo (EM) teniendo como corolario fundamental la ecuación cuántica de Dirac.

Con ello, aquí se propone todo un programa de unificación en el cual el electromagnetismo quiral juega el rol central. La TTS se deriva de las ecuaciones originales de Einstein-Hilbert $G_{\mu v}=k T_{\mu v}$, donde el tensor de Einstein no se modifica. El tensor EM en cambio es quiral y la masa de las partículas es de naturaleza electromagnética. Para el caso del electrón se tiene como consecuencia que, por primera vez, se obtiene la ecuación de Dirac a partir de ondas EM con el campo eléctrico paralelo espacialmente al campo magnético. Como modelo del universo se propone una interfaz o membrana de separación donde ocurren solamente eventos cuánticos. Hay dos regiones enantioméricas de un universo cerrado, o un universo derecho y un universo izquierdo, relacionados por un elemento de simetría PCT (paridad, carga, tiempo) a lo largo de la interfaz. Las ecuaciones de Einstein-Hilbert son estudiadas bajo el enfoque quiral y se discute la electrodinámica quiral y la gravedad en la era de Planck.
\end{abstract}

Palabras clave: Unificación, electrodinámica quiral, era de Planck.

\begin{abstract}
An alternative to the theory of quantum gravity, not yet discovered, is the Theory Simplified Total (TTS) proposal, which aims to unify gravity with the EM taking as a corollary essential quantum Dirac equation.

Thus, this article proposes a whole program of unification in which electromagnetism chiral plays the main role. TTS is derived from the original equations of Einstein-Hilbert $G_{\mu v}=k T_{\mu v}$, where the Einstein tensor is unchanged. The EM tensor instead is chiral and the mass of the particles is electromagnetic nature. In the case of the electron the consequence of this is that, for the first time, Dirac's equation is obtained from EM waves with the electric field spatially parallel to the magnetic field. As a model of the universe an interface or membrane separation is proposed as the only location for quantum events. There are two enanciometrics regions in a closed universe, or right and left universe, connected by an element of PCT (parity, charge, time) symmetry along the interface. Einstein-Hilbert equations are studied under the chiral approach and discusses the chiral electrodynamics and gravity in Planch's era are discussed.
\end{abstract}

Keywords: Unification, chiral electrodynamics, age Planck.

\section{INTRODUCCIÓN}

No es difícil mostrar, sin lugar a dudas, que la física se lleva el honor de ser la disciplina con los mayores adelantos teóricos y con las más grandes aplicaciones tecnológicas. Hoy, a comienzos del siglo XXI, la física sigue el rumbo y la impronta marcada por los grandes progresos logrados el siglo pasado. Como un logro no alcanzado se destaca la formulación de una teoría unificada de todas las fuerzas de la naturaleza. Físicos teóricos de todo el mundo, en solitario o en equipo, han dedicado y dedican una enorme cantidad de tiempo y esfuerzo para la consecución de ese sueño.

1 Instituto de Alta Investigación. Universidad de Tarapacá. Antofagasta № 1520. Arica, Chile. E-mail: htorres@uta.cl 
Al mencionar este esfuerzo, en la búsqueda de la unificación, siempre conviene recordar los trabajos pioneros de Albert Einstein (1879-1955), quien se convirtió en el líder de un sueño singular de hacerlo realidad: la teoría geométrica para la unificación de los campos gravitacionales y electromagnéticos (EM).

El programa de Einstein para la unificación no tuvo éxito por el hecho de no considerar la existencia de campos eléctricos y magnéticos paralelos en el espacio y desfasados en el tiempo pues la existencia teórica y experimental de dichas ondas se ha verificado solo en los últimos años.

A fin de proporcionar una visión global, se ha dividido este trabajo en tres partes. En la primera, se hace una introducción a la electrodinámica quiral (EQ), en la segunda se describen sintéticamente las ideas acerca de la Teoría General de la Relatividad (TGR), los trabajos de Einstein y otros autores para la unificación de los campos, y en la tercera parte se propone una teoría de unificación basada en la electrodinámica quiral para un universo de cuatro dimensiones que da lugar a la Teoría Total Simplificada (TTS).

\section{INTRODUCCIÓN A LA QUIRALIDAD}

Con el avance en la construcción de compuestos artificiales, los materiales quirales han asumido una gran importancia tecnológica en antenas, circuitos de alta frecuencia y en fibras ópticas. Tales materiales, que no tienen la simetría tipo espejo, también se encuentran en la naturaleza desde las galaxias en espiral a moléculas tipo hélices como el DNA, que son ópticamente activas, las cuales muestran birrefringencia a frecuencias ópticas y de microondas. Ya que la quiralidad es un concepto geométrico, es posible concebir la fabricación de materiales quirales artificiales y metamateriales, con aplicaciones en ingeniería electromagnética.

El fenómeno de la actividad óptica, en ciertas substancias biológicas y materiales, fue descubierto por Pasteur (1848-1850) interpretando las observaciones de arreglos asimétricos de átomos dentro de un material ópticamente activo, por lo que se tiene una imagen espejo no superpuesta, arreglo definido como "maniobrable" o quiral.

Con los avances en la teoría de campos electromagnéticos el campo de la esteroquímica se expande fuertemente y se conocen detalles de la estructura de las moléculas y, además, de la naturaleza de estructuras moleculares indispensables para la vida.
Un medio quiral está caracterizado por su maniobrabilidad en su microestructura, ya sea a la izquierda o a la derecha. Esto resulta en un medio quiral polarizado circularmente a la izquierda (LCP) o a la derecha (RCP) y los campos se propagan a diferentes velocidades de fase: el campo con esta última polarización viaja, a través de un medio manipulado a la derecha, más rápido que un campo circularmente polarizado a la izquierda, y viceversa. La actividad óptica, la cual se encuentra en una serie de moléculas orgánicas a frecuencias ópticas, es una manifestación de la quiralidad nativa de estas moléculas. Se observan fenómenos similares al dicroísmo circular (CD) y a la dispersión óptica rotatoria (ORD), con absorción diferencial de las ondas polarizadas circularmente a la izquierda o a la derecha al interior del medio quiral.

Estudios giroscópicos tienen tal riqueza de información que se puede decir que "la actividad óptica entrega una ventana hacia el interior de la fábrica del universo". Los átomos son ahora considerados quirales debido a la débil violación de paridad de la corriente neutral de interacción entre el núcleo y los electrones; la pequeña actividad óptica resultante para los átomos ha hecho más concordante la teoría con la práctica. Los avances tecnológicos en las décadas de los 80-90 han hecho posible la detección de asimetría quiral en dispersión Raman.

Aunque han sido estudiados muchos aspectos de mecánica cuántica, la quiralidad ha sido poco estudiada, falta un estudio sistemático de la teoría clásica de campos electromagnéticos considerando la quiralidad. Con los avances en la ciencia de los polímeros (dieléctricos quirales activos a frecuencias milimétricas, por ejemplo), hacen necesario revisar todos los aspectos relacionales de la teoría de campos electromagnéticos.

Además, a causa de que la quiralidad es un atributo geométrico específico, el conocimiento recogido del estudio de la estructura molecular debe trasladarse al diseño y manufactura de medios quirales artificiales, los cuales deben exhibir CD y ORD a frecuencias de telecomunicaciones. Sólo en el rango intermedio de frecuencia la quiralidad molecular no desaparece cuando se efectúa una transición desde escala microscópica a macroscópica en teoría electromagnética. Lo anterior significa que es posible la construcción de medios artificiales introduciendo objetos quirales micrométricos en un medio huésped aquiral. En un rango de frecuencias intermedias, la microestructura podría tener una dimensión adecuada (2-5\%) respecto de la longitud de onda del material huésped (substrato); consecuentemente, el medio compuesto podría comportarse como efectivamente quiral. La factibilidad de esta idea ha significado un intenso estudio sobre la propagación de ondas electromagnéticas en medios quirales. 
A modo de ejemplo, el ojo humano contiene dos tipos de estructura activa para distinguir entre proceso fotópico (umbral alto) y proceso escotópico (umbral bajo) que son: el bastón y los conos, respectivamente. Varias partes en el ojo son anisotrópicas; en particular las fibras de la retina son algo uniaxiales. Se puede usar la anisotropía estructural para explicar la diferencia en la sensibilidad de los ojos a la luz polarizada circularmente a la derecha y a la izquierda. Así, cualquier tratamiento de la física del ojo no sólo debe incluir anisotropía debido a la microestructura, bastones, conos, ganglios, etc., sino que debería también considerar la macroestructura, es decir, la helicidad de los componentes moleculares cuyas dimensiones pueden ser una fracción significativa de la longitud de onda óptica. Esto será relevante en futuros sistemas ópticos.

La actividad óptica puede ser explicada por la sustitución directa de nuevas relaciones constitutivas en las ecuaciones de Maxwell, es decir, $\mathrm{D}=\varepsilon \mathrm{E}+T \varepsilon \nabla \mathrm{xE}$ y $B=\mu \mathrm{H}+T \mu \nabla \times \mathrm{H}$. Aquí $\varepsilon$ y $\mu$ son la permitividad y permeabilidad respectivamente, mientras $T$ es el parámetro con dimensión de longitud y es el resultado directo de cualquier quiralidad en la manoestructura del medio. Como tal, las ecuaciones constitutivas quirales son aplicables a cualquier región del espectro electromagnético como radiación. En óptica, hasta muy recientemente, sólo fue posible realizar mediciones de intensidad, es decir, de la magnitud pero no de la fase. Así la literatura sobre actividad óptica está relacionada, generalmente, sólo con la diferencia en la intensidad de la luz dispersada cuando un volumen quiral es irradiado ya sea por una onda plana LCP o RCP. Esto significa que sólo mediciones de $\left(n_{L}-n_{R}\right)$ están disponibles, donde $n_{L}$ y $n_{R}$ son los índices de refracción para las ondas LCP y $\mathrm{RCP}$ respectivamente. Aunque cada uno de estos índices de refracción puede estar muy relacionado a $\varepsilon, \mu$, $T$, el conocimiento de $\left(n_{L}-n_{R}\right)$ no es suficiente para inferir los valores de los parámetros constitutivos.

Cuando uno considera este fenómeno a un rango de frecuencias mucho más bajo, $0.5-100 \mathrm{GHz}$, se podría estar más interesado en $\varepsilon, \mu, T$ que en $n_{L}$ y $n_{R}$. Con el advenimiento de los analizadores vectoriales de redes ahora ha llegado a ser posible realizar mediciones muy exactas de magnitud y fase, pero la generación de ondas circularmente polarizadas requiere de tecnología de punta y se prevén aplicaciones en antenas de polarización circular en sistemas GPS de última generación y en comunicaciones satelitales.

El uso del analizador vectorial de redes, en conjunto con experimentos canónicos deseablemente definidos, puede entonces facilitar la medición de $T$. Investigaciones recientes indican que valores de $T$. para compuestos quirales artificiales, en el rango de 8-40 GHz, estarán luego disponibles. Nuestro trabajo específico sobre teoría y simulaciones de la electrodinámica quiral aplicada a solitones, fibras ópticas, sistemas de microondas, sistemas biológicos, etc. están relacionados en [1-18].

\section{EL PROGRAMA DE EINSTEIN PARA LA UNIFICACIÓN}

La historia muestra que la idea de la unificación de las fuerzas de la naturaleza no se origina con los trabajos de Einstein; ni la propuesta de unificación a altas dimensiones tampoco es original de Kaluza [19].

Con el surgimiento de la Relatividad Especial en 1905, casi de inmediato los físicos llegaron a reconocer la invariancia lorentziana en la teoría de Maxwell; y dada la geometría de Minkowski se tornó claro para Einstein y D. Hilbert que la unificación implicaba, de algún modo, la unificación del espacio tridimensional y el tiempo en un "espacio-tiempo continuo" cuadridimensional. En 1915, Hilbert presentó por primera vez una teoría de campo unificado basado en los primeros trabajos de Einstein (1914) sobre la teoría relativista de la gravitación y en los artículos de G. Mie (1912) sobre la electrodinámica no lineal de la materia.

En el trabajo de Hilbert se obtienen las ecuaciones de Euler-Lagrange, derivadas de un principio variacional. Cinco días después de la conferencia de Hilbert sobre su teoría de unificación, Einstein publicó su TGR verificable y verificada que vinculara directamente la distribución y movimiento de materia a la geometría del espaciotiempo.

En la TGR, Einstein geometriza la gravitación en el sentido de que toda la información acerca de las interacciones gravitacionales está contenida en el elemento de línea del espacio-tiempo. La poderosa y bella descripción de la gravitación entusiasmó a los físicos y matemáticos a intentar una geometrización para la unificación con el electromagnetismo (EM).

La búsqueda de una teoría geométrica y unificada de los campos gravitacionales y electromagnéticos ocupó un rol dominante en los últimos veinte años de la actividad científica de Einstein.

Las ideas principales de Einstein para la unificación clásica de las interacciones eran: geometrizar el electromagnetismo, unificar las variables básicas de la 
gravitación y el electromagnetismo en un único objeto geométrico y obtener las ecuaciones de campos unificados a partir de un principio variacional.

Einstein esperaba que las propiedades fundamentales de las partículas elementales y sus respectivos comportamientos cuánticos pudieran ser de algún modo descritos y explicados en el marco de una teoría clásica puramente geométrica.

La teoría general de la relatividad (TGR 1915) está basada en dos objetos geométricos fundamentales: un tensor métrico g y una conexión lineal $\Gamma$. La métrica es necesaria para medir distancias, intervalos de tiempos, velocidades relativas y ángulos. La conexión basada en la noción de transporte paralelo de Levi-Civita sirve a su vez para comparar direcciones, fuerzas y campos en puntos separados en el espacio-tiempo de Riemann.

Todos los intentos iniciales en unificación estaban basados en los objetos geométricos antes mencionados. La idea básica es obtener nuevos grados de libertad en la TGR para describir el electromagnetismo relajando o imponiendo restricciones sobre el tensor métrico $(\mathrm{g})$ y/o $\Gamma$, o incrementar el número de dimensiones de la variedad riemanniana.

Dos años después de que Einstein postulara la TGR, H. Weyl (discípulo de Hilbert) propone un modelo geométrico de la gravitación y del electromagnetismo. Weyl consideró que la geometrización podría ser generalizada a otras fuerzas de la naturaleza. Así, propuso una conexión general dependiente de la trayectoria cuando se compara la longitud de vectores en diferentes puntos del espaciotiempo. En otras palabras, él notó que la TGR está basada en la "relatividad de la dirección" y propuso extenderla a fin de tomar en cuenta la "relatividad de las magnitudes" al permitir una transformación conforme de la métrica.

Esta idea, que llegó a ser conocida como teoría de calibre o de "gauge", no prosperó porque llegaba a contradecir la escala absoluta de masas del mundo real. A pesar del fracaso Weyl reinterpretó los calibres (gauge en el contexto de la física cuántica) al indicar que podrían actuar en las funciones de onda de las partículas cargadas más bien que sobre g. Esta idea inspiró a las teorías de calibre no abelianas y a la interpretación de los potenciales electromagnéticos y de Yang-Mills como conexiones en fibrados principales. También llegó a constituirse en el germen para el desarrollo de las llamadas "teorías gauge de la gravedad".

En 1919, T. Kaluza propuso una teoría de la gravitación de cinco dimensiones. Esta idea fue trabajada por Einstein y colaboradores en 1923. Einstein vuelve sobre esta teoría pentadimensional en un trabajo publicado en 1927; también lo hace en cuatro trabajos $(1931,1932,1938$ y 1944) con sus colaboradores, pero sin llegar a geometrizar el EM ni a la unificación de los campos gravitacional y EM. A. Eddington propuso considerar a $\Gamma$ como la cantidad básica de la TGR y derivar de ella tanto el tensor métrico como el campo electromagnético al dividir el tensor de Ricci, $R_{\mu \nu}$ en sus partes simétricas y antisimétricas. Einstein, en cinco trabajos (cuatro en 1923 y uno en 1925), desarrolló esta idea al postular que la densidad lagrangiana debería ser proporcional a la cantidad |det $R_{\mu v} \mid 1 / 2$. Desafortunadamente, todos estos intentos llevaron a ecuaciones incompatibles con los experimentos y Einstein se vio obligado a abandonar este camino. En 1925 Einstein consideró una teoría basada en la conexión $\Gamma$ y una g ${ }^{\mu \nu}$ no simétrica e identificaba a $\mathrm{g}^{[\mu v]}$ (la parte antisimétrica de $\mathrm{g}^{\mu v}$ ) con el campo electromagnético; volvió sobre esta idea en los últimos años de su vida trabajando en una "teoría asimétrica" fundamentada en la métrica y en la conexión. Sobre esta línea de investigación publicó 11 trabajos entre 1925 y 1955. Bajo la influencia de Cartan, Einstein genera una nueva línea de investigación en la que se dota a la variedad del espacio-tiempo de una nueva entidad geométrica llamada torsión inventada por Cartan en 1922.

Este esquema fue transformado por un nuevo y poderoso concepto geométrico llamado teleparalelismo, también desarrollado por Cartan. Teleparalelismo significa que la curvatura total es cero, o una suposición más débil: que el tensor total de Ricci es cero. Estas ideas fueron trabajadas por Einstein y publicadas en tres trabajos (uno en 1929 y dos en 1930). En esa área tampoco logró la ansiada unificación. Las teorías de unificación basadas en teleparalelismo han sido reconsideradas en años recientes siguiendo el enfoque de la geometría diferencial moderna [20].

En resumen, Einstein se embarcó en un programa geométrico de unificación de las interacciones clásicas gravitacionales y electromagnéticas en más de cuarenta trabajos. A pesar del fracaso, aun así entreabrió nuevas sendas hacia la búsqueda de la unificación de las fuerzas de la naturaleza, en cuya tarea se han ocupado importantes físicos durante el siglo XX.

Pero la mayoría de estos esfuerzos están bajo el enfoque de la teoría cuántica de campos. De hecho, las tres cuartas partes de las fuerzas de la naturaleza conocidas son estudiadas en el marco de la mecánica cuántica; y ya se ha logrado la unificación de las fuerzas débiles con las electromagnéticas. La unificación con la fuerte en este esquema no debe tardar en concretarse. La gravitación, por 
su naturaleza misma, se resiste a entrar en ese esquema. Existen por lo menos cuatro programas de cuantización del campo gravitatorio [21], todos los cuales parecen están destinados al fracaso. El más popular de estos fracasados esfuerzos es el programa de las "variables de Asthekar", físico hindú que pretende cuantizar el campo gravitatorio "a la canónica", es decir, siguiendo el mismo procedimiento que llevó a la cuantización del campo EM. El punto crucial es que si deseamos cuantizar el campo gravitacional deberíamos, como bien han señalado R. Penrose y R. Wald [21], reconstruir la mecánica cuántica sobre nuevos fundamentos.

El otro programa de unificación vía geometría diferencial es también extraordinariamente difícil. Es pertinente mencionar que en esta área de trabajo se distinguen dos líneas de investigación. Una en la que se mezclan conceptos de calibres de la mecánica cuántica con conceptos y herramientas de la topología diferencial. Un ejemplo de ello son las llamadas teorías de Kaluza-Klein, en cinco o más dimensiones. La otra línea de acción proviene de los trabajos de Wheeler, quien partiendo del enfoque de una geometría diferencial pura ha publicado la más elaborada geometrización del electromagnetismo de toda la literatura [20].

\section{MODELO QUIRAL DEL UNIVERSO}

Einstein en su visión del universo y en su programa de unificación, aun teniendo presente el origen cuántico de la materia, no pudo concretar la unificación GEM. Tal vez el recorrido zigzagueante de Einstein en su programa de unificación fue producto de las numerosas tentativas de modificar el lado izquierdo de su ecuación $G_{\mu \nu} k T_{\mu v}$, dejando el tensor de materia $T_{\mu \nu}$ sin alterar. En la TTS lo que cambia es el tensor $T_{\mu v}$. Conviene aquí decir algo al respecto de la relatividad general y la mecánica cuántica.

En la actualidad, no hay duda de que la teoría de la relatividad y el modelo del Big-Bang son exitosos a la hora de presentarnos un panorama general de cómo el universo que hoy disfrutamos es consecuencia de la evolución bajo ciertas condiciones iniciales del universo que había luego de unas cuantas fracciones de segundo y de la aplicación de leyes conocidas de la física. Insistimos, no es que se conozcan todas las respuestas ni todos los detalles, sino que el modelo brinda la plataforma sobre la cual estas preguntas y estos detalles pueden ser bien planteados y abordados con la estrategia de las ciencias físicas.

La relatividad de Einstein permanecerá como una portentosa contribución de la ciencia del siglo XX y un formidable tributo al ingenio humano. Tanto en su versión especial como en la general cuando haya materia que curve el espacio-tiempo, la relatividad será una poderosa herramienta de interpretación de una parte de la realidad física.

La revolución iniciada por la relatividad cambió de manera contundente la forma como debemos entender al espacio, al tiempo y a la materia. Nos brinda una imagen más coherente y unificada del mundo físico: la manera por la que brillan las estrellas tiene que ver con el retraso de relojes en movimiento. Entendemos mejor por qué cierta escala el sistema newtoniano da tan buenos resultados. Una buena parte de sus predicciones han sido corroboradas dándole sentido a las observaciones.

Otras, como la existencia de ondas gravitatorias, nos permitirán 'mirar' el universo con otra mirada, más profunda, que habrá de revelarnos mucho acerca del universo en que vivimos. La 'flexibilidad' del tiempo y el espacio permite considerar las seductoras posibilidades de desaparición del tiempo como en los agujeros negros, la aparición del tiempo en el Big Bang, la expansión del espacio a escala cosmológica, que en la rígida perspectiva newtoniana eran impensables.

Sin embargo, sabemos que algo importante está faltando. Las dos grandes revoluciones del siglo XX, la relatividad general y la cuántica, son incompatibles ente sí. Cada una es exitosa en su ámbito: la teoría cuántica describiendo el micromundo y la relatividad general, el cosmos a gran escala. Usan estrategias diferentes, imágenes de la realidad diferentes, metáforas diferentes y métodos matemáticos diferentes. La relatividad elude la naturaleza cuántica y la teoría cuántica elude el espacio-tiempo curvo. La primera no acepta el principio de incertidumbre y la segunda no acepta el principio de equivalencia. Para la relatividad general, la constante de Planck $h$ es igual a cero; para la teoría cuántica, la constante, gravitacional de Newton $G$ es igual a cero.

Obviamente ambas son aproximaciones. La construcción de una teoría cuántica de la gravitación de la cual obtengamos casos límites apropiados, a la teoría cuántica de campos y a la relatividad, es la parte faltante de la revolución de la física del siglo XX, y es tarea pendiente para la física del nuevo milenio. Únicamente con esta teoría en la mano podremos entender qué ocurre cuando lo muy pequeño pero muy pesado aparecen en la misma situación física. Tan sólo con una teoría cuántica de la gravedad podremos hablar con propiedad de la naturaleza del Big Bang o de la singularidad escondida en el centro de los agujeros negros. 
Los intentos y acercamientos a esa(s) teoría(s) sugieren que en la llamada escala de Planck en tiempo y espacio $\left(L_{P} \approx 10^{-33} \mathrm{~cm}, \mathrm{tp} \approx 10^{-43} \mathrm{seg}\right.$ ), característica de los fenómenos cuánticos gravitacionales, la naturaleza del espacio y el tiempo es radicalmente distinta de lo que observamos, tal vez cambie el número de dimensiones del espacio. Lo importante, como siempre, estará en las consecuencias y predicciones que una presunta teoría cuántica de la gravedad proponga, y que nos permita entender un poco mejor el universo que nos alberga, y tal vez un poco mejor a nosotros mismos.

Una alternativa a la teoría cuántica de la gravedad, aún no descubierta, es la teoría propuesta en los artículos en esta edición de Ingeniare, la Teoría Total Simplificada (TTS) que postula unificar la gravedad con el EM teniendo como corolario fundamental la ecuación cuántica de Dirac. Ver figura 1.

Con ello, aquí se propone todo un programa de unificación en el cual el electromagnetismo quiral juega el rol central
[22]. La TTS se deriva de las ecuaciones originales de Einstein-Hilbert $G_{\mu \nu}=k T_{\mu v}$, donde el tensor de Einstein no se modifica. El tensor EM en cambio es quiral y la masa de las partículas es de naturaleza electromagnética. Para el caso del electrón se tiene como consecuencia que por primera vez se obtiene la ecuación de Dirac a partir de ondas EM con el campo eléctrico paralelo espacialmente al campo magnético [22-26].

En la figura 1 se muestra una interfaz o membrana de separación donde ocurren solamente eventos y sucesos cuánticos.

Hay dos regiones enantioméricas de un universo cerrado, o un universo derecho y un universo izquierdo, relacionados por un elemento de simetría PCT (paridad, carga, tiempo) a lo largo de la interfaz. Las características principales de ambas regiones enantioméricas están definidas en la figura y representan un modelo con todos los atributos requeridos por un vacío teórico. Lejos de la membrana de separación son válidas las ecuaciones de Einstein-

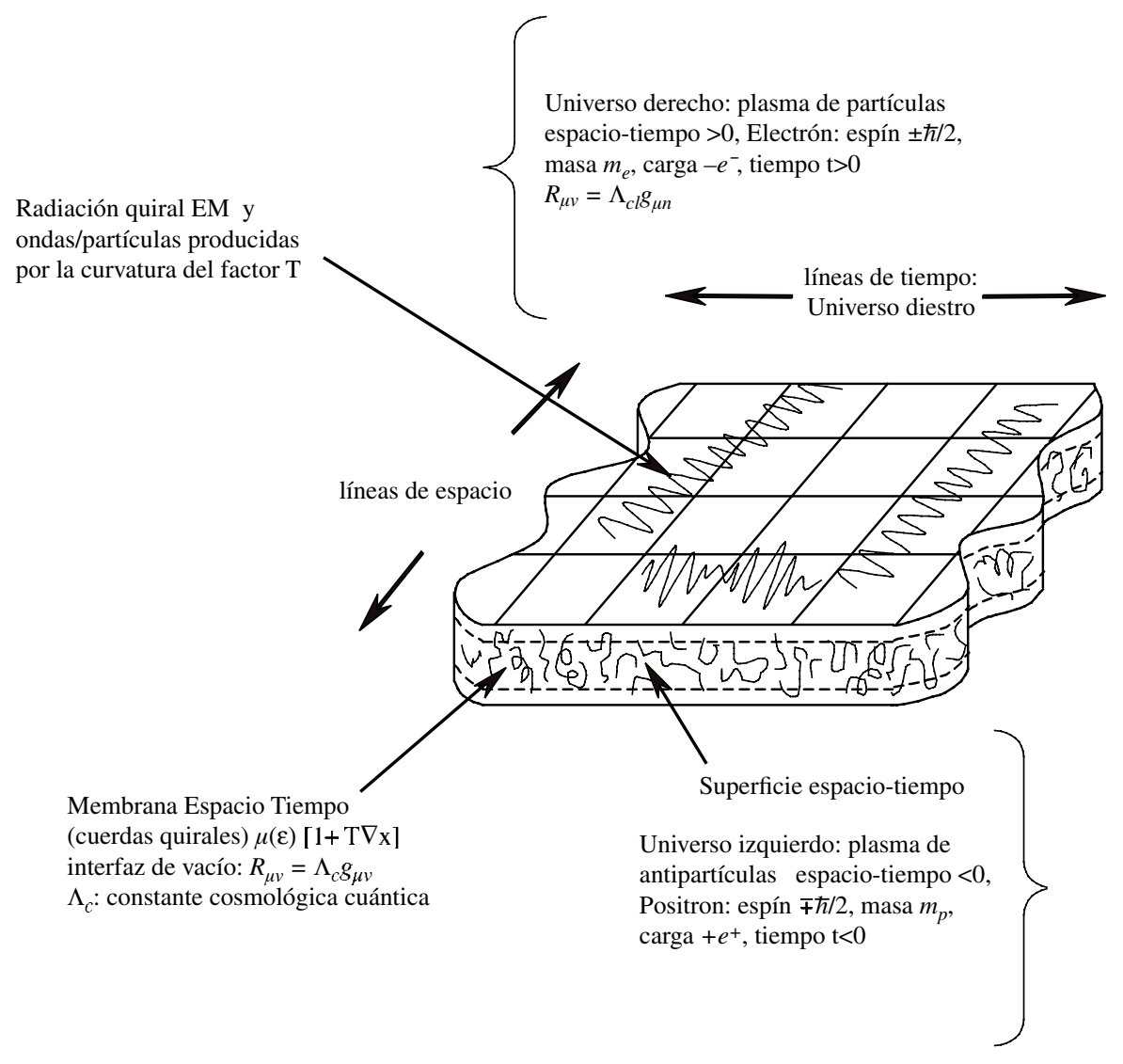

Figura 1. Modelo del universo con dos regiones enantioméricas separadas por una membrana cuántica con $\Lambda_{\mathrm{c}} / \Lambda_{\mathrm{cl}}$ del orden de $10^{120}$. 
Hilbert $G_{\mu v}=k T_{\mu v}$, que son de naturaleza clásica. En la vecindad de la capa de separación hay efectos cuánticos y es válida la ecuación de Dirac para partículas en la región enantiomérica diestra y para antipartículas en la otra región [22-26].

Cada región puede ser considerada como un vacío, tiene una constante cosmológica $\Lambda_{c l}$, que tiende a cero, la membrana posee una constante cosmológica de naturaleza cuántica tal que $\Lambda_{c} / \Lambda_{c l} \sim 10^{120}$.

\section{ECUACIONES EINSTEIN-HILBERT BAJO EL ENFOQUE QUIRAL}

Desde los estudios de J. Earman y C. Glymour [27] se hizo evidente que las ecuaciones de la relatividad general de Einstein se descubrieron casi simultáneamente, pero con diferentes métodos, por D. Hilbert y A. Einstein [28-30].

Según el actual punto de vista Einstein y Hilbert, de forma independiente uno de otro y de diferentes maneras, descubrieron las ecuaciones del campo gravitacional [30]. En el trabajo de Einstein con $\Lambda_{c l}=0$ las ecuaciones de campo gravitacional son dadas por:

$$
\sqrt{-g} R_{\mu v}=-\kappa\left(T_{\mu v}-g_{\mu v} T\right)
$$

Donde, como de costumbre, $g_{\mu \nu}$ es un tensor métrico; $R_{\mu \nu}$ es el tensor de Ricci, $k$ es la constante gravitacional de acoplamiento, $T_{\mu \nu}$ es el tensor de densidad de energía-momento para la materia, $T$ es la traza de $T_{\mu \nu}$ : $T=g^{\mu v} T_{\mu v}$ Hilbert, habiendo visto el término "traza" $\frac{1}{2} g_{\mu v} T$, también
lo introdujo en sus ecuaciones,

$$
\sqrt{g}\left(R_{\mu \nu}-g_{\mu v} R\right)=-\frac{\partial \sqrt{g} L}{\partial g^{\mu \nu}}
$$
el término traza (en este caso $\frac{1}{2} g_{\mu v} R$, donde la traza
$R=g^{\mu v} R_{\mu v}$ ).

El enfoque de Hilbert es exacto donde todo es definido por la función lagrangiana

$$
H=R+L
$$

Descubierta por Hilbert, que desempeña un papel clave para la obtención de las ecuaciones de gravitación en el marco del principio de mínima acción [32]. Pero fue Einstein sobre la base de la idea de la equivalencia de la aceleración y la gravedad que formula la ley de conservación general de la energía-impulso [31].

A través del principio de mínima acción (Axioma I de Hilbert) y de la teoría de invariantes es que en este artículo se considera un modelo quiral para el universo considerando no la electrodinámica de Mie sino la EQ.

Vamos a examinar con atención el enfoque de Hilbert [32]. El Axioma I es tal que:

Las leyes de la física son los eventos definidos por la función mundo $H$ cuyos argumentos son

$$
\begin{gathered}
g_{\mu v}, \quad g_{\mu v l}=\frac{\partial g_{\mu v}}{\partial x^{l}}, \quad g_{\mu v l k}=\frac{\partial^{2} g_{\mu v}}{\partial x^{l} \partial x^{k}} \\
q_{s}, \quad q_{s l}=\frac{\partial q_{s}}{\partial x^{l}}, \quad(l, s=1,2,3,4)
\end{gathered}
$$

Siendo que la variación de la integral

$$
\int H \sqrt{g} d \omega
$$

con

$$
\left(g=\left|g_{\mu v}\right|, d \omega=d x_{1} d x_{2} d x_{3} d x_{4}\right)
$$

es cero para cualquiera de los 14 potenciales $g_{\mu v}, q_{S}$. Bajo el enfoque quiral la variación temporal $\partial / \partial t$ pasa a ser $\partial / \partial t \rightarrow(1+T \nabla \mathrm{x}) \partial / \partial t$.

En cuanto a la función mundo, de acuerdo a Hilbert, axiomas adicionales son necesarios para su definición no ambigua. Si sólo las segundas derivadas de los potenciales pueden entrar en las ecuaciones de la gravitación, esto no cambia con EQ la función tiene que tener la forma

$$
H=R+L_{Q}
$$

Donde $R$ es un invariante del tensor de Riemann (curvatura escalar de un sistema múltiple de cuatro dimensiones):

$$
R=g^{\mu v} R_{\mu v}
$$




$$
R_{\mu v}=\partial_{v} \Gamma_{\mu \alpha}^{\alpha}-\partial_{\alpha} \Gamma_{\mu v}^{\alpha}+\Gamma_{\mu \alpha}^{\lambda} \Gamma_{\lambda v}^{\alpha}-\Gamma_{\mu v}^{\lambda} \Gamma_{\lambda \alpha}^{\alpha}
$$

$L_{Q}$ es una lagrangiana quiral que es función de las variables $g^{\mu \nu}, g_{\ell}^{\mu \nu}, q_{s}, q_{s k}, T$ El factor quiral $\mathrm{T}>\mathrm{o}$ corresponde a la región enantiomérica diestra o nuestro universo de materia, $\mathrm{T}<0$ a la región izquierda (ver figura 1 del modelo para el universo) y $R_{\mu \nu}$ es el tensor de Ricci. Además de eso, suponemos más adelante que $L_{Q}$ no depende de $g_{\ell}^{\mu v}$.

A partir de la variación en los 10 potenciales gravitacionales se tienen 10 ecuaciones diferenciales de Lagrange:

$$
\frac{\partial \sqrt{g} R}{\partial g^{\mu \nu}}-\partial_{k} \frac{\partial \sqrt{g} R}{\partial g_{k}^{\mu \nu}}+\partial_{k} \partial_{l} \frac{\partial \sqrt{g} R}{\partial g_{k l}^{\mu \nu}}=-\frac{\partial \sqrt{g} L_{Q}}{\partial g^{\mu \nu}}
$$

Es fácil de ver de las ecuaciones anteriores que $R$ y $R_{\mu v}$ que son derivadas de segundo orden entran en la métrica linealmente. Todos los demás tensores se obtienen como combinaciones de estos tensores con similares propiedades.

Así, la ecuación de campo gravitacional sometido al tensor electromagnético quiral es

$$
\left[\begin{array}{ll}
\sqrt{g} & R
\end{array}\right]_{\mu v}=-\kappa \stackrel{Q}{T}_{\mu v}
$$

De aquí la ley de conservación covariante de la energíaimpulso se deduce naturalmente. El tensor densidad $\left[\begin{array}{ll}\sqrt{g} & R\end{array}\right]_{\mu v}$ contiene por construcción las derivadas lineales de segundo orden.

Las ecuaciones de Lagrange, de Hilbert bajo el enfoque quiral son consecuencia del principio de mínima acción (Axioma I de Hilbert). Así las ecuaciones de la gravitación tienen la forma:

$$
\sqrt{g}\left(R_{\mu \nu}-\frac{1}{2} g_{\mu \nu} R\right)=\left[\begin{array}{ll}
\sqrt{g} & R
\end{array}\right]_{\mu v}=-\stackrel{Q}{T_{\mu \nu}} .
$$

Se elige el invariante $L_{Q}$ en función de las variables $g^{\mu v}, q_{\sigma}, \partial_{v} q_{\sigma}, T$, por lo que se obtienen las ecuaciones generalizadas de Maxwell

$$
\left[\begin{array}{ll}
\sqrt{g} & L_{Q}
\end{array}\right]^{v}=0
$$

Luego, sobre la base del Teorema II, se tiene que la función de Lagrange depende de las derivadas del potencial $q_{v}$, sólo a través del tensor $F_{\mu v}$, es decir,

$$
L_{Q}\left(F_{\mu v}\right)
$$

Donde

$$
F_{\mu v}=\partial_{\mu} q_{v}-\partial_{v} q_{\mu}
$$

Según el teorema II las cuatro identidades tienen lugar en el invariante $L_{Q}$ :

$$
\nabla_{\mu} \stackrel{Q}{T_{v}^{\mu}}=F_{\mu v}\left[\begin{array}{ll}
\sqrt{g} & L_{Q}
\end{array}\right]^{\mu}+q_{v} \partial_{\mu}\left[\begin{array}{ll}
\sqrt{g} & L_{Q}
\end{array}\right]^{\mu}
$$

Se desprende de la identidad (17) que, en caso de las ecuaciones de movimiento de un sistema material (14) se sostienen, entonces la ley de la conservación covariante se lleva a cabo:

$$
\nabla_{\mu} \stackrel{Q}{T_{v}^{\mu}}=0
$$

Si uno hace uso de las ecuaciones de la gravitación para la identidad (17) se obtiene de la misma manera que de las ecuaciones de Hilbert [31, 32]:

$$
F_{\mu v}\left[\begin{array}{ll}
\sqrt{g} & L_{Q}
\end{array}\right]^{\mu}+q_{v} \partial_{\mu}\left[\begin{array}{ll}
\sqrt{g} & L_{Q}
\end{array}\right]^{\mu}=0
$$

Ecuaciones (19) tienen que ser compatibles con las ecuaciones, que se deriven del principio de mínima acción con la misma lagrangeana $L_{Q}$. Sólo es posible en el caso de que "ecuaciones generalizadas de Maxwell" se autosustentan:

$$
\left[\begin{array}{ll}
\sqrt{g} & L_{Q}
\end{array}\right]^{v}=0
$$

En el caso particular de que $L_{Q}=\alpha_{Q} I$. El segundo término en Eq. (19) desaparece idénticamente y se llega a las ecuaciones

$$
F_{\mu v}\left[\sqrt{g} L_{Q}\right]^{\mu}=0
$$

De ello se deduce, por lo tanto, que si el determinante $\left|F_{\mu \nu}\right|$ no es cero, se tienen las ecuaciones de Maxwell $\left[\begin{array}{ll}\sqrt{g} & L_{Q}\end{array}\right]^{\mu}=0$. Esto está plenamente de acuerdo con el principio de mínima acción (Axioma I de Hilbert). De esta forma, la ecuación de Maxwell son consecuencia de las ecuaciones de gravedad y de las cuatro identidades 
(21). Cabe señalar que originalmente Hilbert obtuvo la ecuación de campo gravitatorio "no de un arbitrario sistema material, sino que en base a la teoría de Mie [33] con un lagrangeano en la forma $L=\alpha Q+f(q)$ donde $\alpha$ es una constante y $f(q)$ es el término no covariante de Mie con

$$
Q=F_{\mu v} F_{\lambda \sigma} g^{\mu \sigma} g^{\nu \lambda}, q=q_{\mu} q_{v} g^{\mu \nu},
$$

Este hecho hace que de dicha teoría no se obtiene una teoría sustentable para el electrón, pero el método de Hilbert es correcto en general, y es un excelente punto de partida para la unificación propuesta en este trabajo.

En las referencias [22-26] se muestra específicamente que la unificación de la gravedad con el EM de Maxwell conocido en aquella época no era sustentable, por cuanto el determinante del tensor de campo de Maxwell siempre es cero. El hecho de que las ecuaciones de la gravitación implican cuatro ecuaciones para el sistema material, hace muy atractivo el método de Hilbert por la sencillez y potencia del mismo, por lo que se presta muy bien al enfoque de la gravitación con electrodinámica quiral.

Basado en los argumentos expuestos en [34-36] y suponiendo que el electromagnetismo quiral nace en la interfaz de vacío (membrana de separación de las dos regiones enantioméricas), y recordando que $\partial / \partial t$ se transforma en $(1+T \nabla \times) \partial / \partial t$, la ecuación (5) de Hilbert reformulada es $(1+T \nabla \times) \partial q_{s} / \partial t$.

Las tensiones electromagnéticas espaciales [34] son de la forma

$$
\varepsilon \rightarrow \varepsilon_{0}\left(1+T \varepsilon_{\mu l v} \frac{\partial}{\partial x_{l}}\right), \mu \rightarrow \mu_{0}\left(1+T \varepsilon_{\mu l v} \frac{\partial}{\partial x_{l}}\right)
$$

Además se tiene que el potencial $q_{s}$ tiene las componentes vectoriales $\boldsymbol{q}=\boldsymbol{A}+T \nabla \times \boldsymbol{A}$, donde $\boldsymbol{A}$ es el potencial vector de Maxwell y el campo magnético en un espacio curvado es $\boldsymbol{B}=\nabla \times \boldsymbol{q}$.

El factor quiral $\mathrm{T}>0$ corresponde a la región enanciométrica diestra donde se tiene un plasma de partículas, $\mathrm{T}<0$ a la región izquierda, donde existe un plasma de antipartículas (ver figura 1 del modelo para el universo). En el apéndice, las ecuaciones de Maxwell derivadas plenamente en forma relativística de $L_{Q}$ son presentadas además de las ecuaciones de onda en régimen quiral. En la siguiente sección se discute esta teoría en los inicios del Big-Bang.

\section{LA ELECTRODINÁMICA QUIRAL Y LA GRAVEDAD EN LA ERA DE PLANCK}

Se sabe que el universo se está expandiendo debido a la oscuridad de la noche. La dinámica dominante del cosmos es, al parecer, una expansión repulsiva de "antigravedad" a gran escala en contraste con la de corto alcance de atracción de la materia en las galaxias y las estrellas.

Este fenómeno es la respuesta a la paradoja de Olber, es decir, el hecho de que el cielo, que se llena de un infinito campo de estrellas y galaxias, no brilla como una estrella sólida, sino que es predominantemente oscuro y tiene sólo la radiación de temperatura de $2,7^{\circ} \mathrm{K}$. El hecho de que el universo se está expandiendo significa que las estrellas y galaxias se alejan entre sí y hay un corrimiento al rojo, por lo que el cielo de la noche es de por sí oscuro y frío, en lugar de ser un campo brillante y caliente de polvo de estrellas. Este hecho permite un universo de baja temperatura donde la vida pueda florecer. Por lo tanto, la expansión del universo puede ser vista como esencial para la vida.

El fenómeno que provoca la expansión acelerada del universo se conoce como "energía oscura" y se puede decir que la causa el vacío. La densidad de la energía oscura puede ser identificada como el término "constante cosmológica" en las ecuaciones de la relatividad general. Este término puede ser entendido a través del concepto de un universo de plasma, donde la electrodinámica cósmica desempeña un papel de igualdad con la gravitación que es la conformación del cosmos y de sus estructuras. De hecho, la gravitación ahora puede ser entendida como una manifestación de la electrodinámica de un gran número de partículas cargadas.

Este término cosmológico puede estudiarse en el contexto de esta teoría TTS que determina el valor de la constante de la gravitación.

La principal hipótesis del universo de plasma es que la electrodinámica desempeña un papel igualitario con la gravedad en la configuración de las estructuras del cosmos. Es posible ampliar este principio incluso a la microescala del cosmos, y considerar la posibilidad de que incluso el vacío en sí mismo puede ser analizado como un "plasma virtual" de partículas cargadas. De esta manera es posible desarrollar un modelo del universo que va desde la longitud de Planck al radio de Hubble para el universo, como un tejido continuo de la electrodinámica, y con los dos límites de longitud que se correlacionan entre sí. 
Esta unificación se basa en dos postulados: el EM se unifica con la gravedad a la longitud de Planck, donde el campo eléctrico $\boldsymbol{E} p$ es paralelo al campo magnético $\boldsymbol{B} p$. El segundo postulado establece que en el tiempo de Planck la gravedad y los campos EM están unificados y que se separaran, y se convierten en diferentes variables a nivel de mesoescala. Aquí encontramos diferencias con [37]. Esta teoría puede predecir campos de gravedad y el valor de la constante de gravedad. Asimismo, se puede predecir el valor del tiempo de Hubble y temperatura de la radiación cósmica de fondo. Según esta teoría, la razón de masa de electrones a protones $R_{m}$ asume un rol central. Sin embargo, es evidente que el valor de $R_{m}$ depende de la fuerza fuerte, y los nucleones, que constituyen la mayor parte de la masa visible del universo, se derivan de los quarks. Esto es esencial para cualquier teoría de unificación [38]. Aquí, en la TTS, la EQ es como una extensión de la teoría de Sakharov para la gravedad y el origen tanto de protones y electrones, pero la unificación nace en la era de Planck con un plasma cosmológico en nuestro universo diestro.

La teoría TTS es un intento de crear una teoría geométrica para resolver el problema de Einstein-Dirac de la unificación de la gravedad y el EM. La teoría por ahora se limita a los protones y electrones. La teoría está todavía en un estado temprano de desarrollo, es decir, que se describe como un "modelo de Bohr" de la unificación, por analogía con la mecánica cuántica y el modelo del átomo de hidrógeno, y se basa en una extensión de los trabajos de Einstein y Kaluza. El aspecto separado de la gravedad y el EM relativos a los protones y electrones proviene desde la escala de Planck y se produce con la aparición de una dimensión quiral como un equivalente de la quinta dimensión de Kaluza-Klein. La teoría TTS comienza con el principio de acción de Hilbert, que permite la obtención de las ecuaciones fundamentales de los campos de vacío con la extremización de la acción integral

$$
H=(16 \pi G)^{-1} \int(R-2 \Lambda) \sqrt{-g} d x^{4}
$$

Donde es la curvatura escalar de Ricci de la relatividad general, $\Lambda$ es la constante cosmológica, $G$ es la constante de la gravitación de Newton. Aquí, la acción integral para un campo cuántico de partículas con espín y masa, $m$ cuando $L_{E M}$ procede de una $\boldsymbol{E} Q$ y $E_{p}=\boldsymbol{i} \boldsymbol{B}_{p}$. Este campo produce un tensor de tensión de la forma $p=p c^{2}$, es decir, una presión negativa, que a su vez impulsa una explosión del cosmos [39, 40]. Si suponemos que el cosmos es una entidad electrodinámica, entonces sería natural que, en un universo en expansión rápida, una especie de reacción de la ley de Lenz se debe haber producido para frenar la expansión cósmica. Esto también puede ser considerado como un "principio cósmico de la mínima acción”. Esta reacción sería la aparición de campos EM y de materia que presentan una densidad de energía positiva que dramáticamente frena la explosión inicial del universo. Este escenario es, de hecho, el escenario inflacionario, que se considera ahora el principal modelo de la cosmología. Sin embargo, puesto que nuestro objetivo final es la modificación tecnológica práctica de la gravedad, vamos a examinar ahora la relación de la gravedad y el EM en detalle.

En la teoría de la relatividad general (RG) de Einstein, la gravedad surge de la geometría del espacio tiempo determinado por las propiedades del tensor métrico $g_{i j}$. El límite newtoniano se recupera donde el espacio no es muy curvado y el potencial de Newton $\phi$ es en realidad parte del elemento diagonal principal del tensor métrico $g_{t t}=-1-2 \phi / c^{2}$. Desde el punto de vista de la TTS, la gravedad surge de la electrodinámica quiral. Sobre la base de esto, parece ser que una buena generalización para modelar la gravedad es que el tensor métrico de la RG es en realidad un tensor EM normalizado. Es decir, los campos EM no sólo son la curvatura de la métrica, sino que la métrica misma. Esto significa que el espacio tiempo es en realidad un vasto mar de radiación ultrapoderoso de EM.

Con el fin de satisfacer los postulados de la TTS debemos tener una generalización covariante y física de campo para el vacío

$$
g_{i j}=4 F_{i k} F_{j}^{k} / F^{\mu v} F_{\mu v}=4 F_{i k} F_{j}^{k} / T_{0}
$$

Donde $F_{i k}$ es el tensor de Faraday y $T_{0}$, es la covariante generalización normalizada del escalar de tensión escalar. La forma de esta expresión para el tensor métrico es determinada por el primer postulado TTS, esto es, para que un campo EM ultrafuerte llene el cosmos el tensor de Maxwell es

$$
T_{i j}=1 / 4 \pi\left(F_{i j} F_{j}^{k}-1 / 4 g_{i j} F^{\mu v} F_{\mu v}\right)
$$

que debe desaparecer en todas partes. Así, en la TTS, un poderoso campo EM determina la geometría del espacio, pero en sí no es detectable directamente, sino que por ser tan poderoso se anula a sí mismo. Cabe señalar que $F_{j}^{k}$, la forma mixta del tensor de Faraday, es a menudo escrito como $g_{i j} F^{i k}$. Físicamente, sin embargo, el objetivo principal de la TTS es demostrar que la gravedad, los campos EM, y, por tanto, la geometría, son unificadas y son partes de una relación cíclica. Esto significa que en la TTS, EQ implica la geometría y viceversa, de 
manera que para empezar con uno en lugar del otro es una cuestión de convención, y para reclamar que uno es superior y más fundamental que el otro no parece razonable. La EQ es dada por

$$
\begin{aligned}
& L_{Q}=\int \kappa g_{\mu v} \sqrt{-g} \boldsymbol{E}_{Q} \cdot \boldsymbol{B}_{Q} d x^{4}, \\
& \boldsymbol{E}_{Q}\left(\boldsymbol{B}_{Q}\right)=(1+T \nabla \times) \boldsymbol{E}(\boldsymbol{B})
\end{aligned}
$$

la densidad de energía neta del espacio puede ser ligeramente negativa con este lagrangeano si $\mathrm{T}$ tiene un valor apropiado. Un mecanismo similar fue propuesto por primera vez en [40] y se denomina “Zel'dovich reacción”. En este artículo se deriva el valor de $G$ como la "elasticidad de la métrica del espacio" con el supuesto de que la longitud de Planck $T_{p}=\left(G \hbar / c^{3}\right)^{1 / 2}$, donde $T_{p}$ es el factor quiral de nuestro R-Universo, $\hbar$ es la constante de Planck. El valor de la integral se determina por la frecuencia de corte cerca de la frecuencia de Planck $\omega_{p}=c / T_{p}$. Numéricamente este valor de $T$ es igual al radio de Planck [39, 40]. Esta conexión entre la gravedad y el campo EQ alienta la posibilidad de que la gravedad pueda un día ser modificada directamente por medios externos.

La manera más sencilla de obtener una energía oscura o la constante cosmológica es permitir que $\mathbf{E} \rightarrow \boldsymbol{i} \boldsymbol{B}$ y esto genera una constante cosmológica y, por tanto, un universo en expansión.

Teniendo en cuenta la hipótesis de un universo de plasma donde domina la electrodinámica quiral, suponemos la existencia de modos quirales que componen la energía oscura. En la escala de Planck, la incertidumbre de Heisenberg permite que se generen masas $M_{p}$, que tienen una longitud de onda de Compton igual a su radio de Schwartzchild

$$
G M_{P} / c^{2}=r_{P}=T_{P}=\hbar / 2 M_{P} c=\sqrt{G \hbar / 2 c^{3}}
$$

A la longitud de Planck, los horizontes de evento de los agujeros negros aparecen y desaparecen en un período de Planck, y la distinción topológica entre estar dentro y fuera de un evento horizonte desaparece. Por breves instantes de un período de Planck, las partículas de antimateria pueden interaccionar con las partículas ordinarias. Esto produce la cuantización de la carga $e=k h c / 2 \pi \mathrm{T}$. Sin embargo, el tamaño de $T$ debe ser del orden de la longitud de Planck, para dar el correcto valor de la carga y las masas son del orden de la masa de Planck. Sin embargo, la existencia de la escala de masas de protones y electrones significa que el tamaño efectivo de la dimensión compacta debe ser mucho mayor, es decir, del orden de la longitud de onda de Compton de un protón o el radio clásico electrónico. Esto puede ser entendido conceptualmente como un "renormalization", efecto debido a la energía negativa de la formación de la dimensión quiral.

La teoría de TTS así permite, por un pequeño aumento de la dimensionalidad, la aparición explícita de los campos EM, y las partículas con carga y masa, para protones y electrones, junto con la gravedad, desde un principio variacional con $\left.\sigma=m_{p} / m_{e}\right)^{1 / 2}=42.85003$.

Esto constituye una descripción muy básica del cosmos como un todo, cuyos principales componentes más conocidos son los protones y electrones. Si se concibe que el principio de la acción asume un campo EQ no masivo, entonces la aparición de la quiralidad permite la captura o la dispersión de quantas no masivos que crean masa en reposo, carga, espín y por lo tanto las partículas. La captura de la energía EM quirales en una dimensión compacta puede ser concebida como una imagen compactada del espacio-tiempo. El tamaño de la dimensión compactada puede considerarse que sea del orden del radio clásico del electrón, $r_{e}=e^{2} / 4 \pi \varepsilon_{0} m_{0} c^{2}$, que es el tamaño aproximado del protón. El carácter de la dimensión compacta debe ser una imagen global del espacio-tiempo, esto significa que el protón, es decir, el espacio, como partícula, debe tener tres subdimensiones para satisfacer la condición de radio clásico y también para la neutralidad del cosmos. Esta combinación es satisfecha por la actual teoría de los quarks, donde el protón está formado por tres quarks que satisfacen $q_{x}=q_{y}=2 e / 3$ y $q_{z}=-e / 3$, es decir,

$$
q_{x}+q_{y}+q_{z}=e(2 / 3+2 / 3-1 / 3)=e
$$

$$
q_{x}^{2}+q_{y}^{2}+q_{z}^{2}=e^{2}(4 / 9+4 / 9+1 / 9)=e^{2}
$$

Donde $q_{x}, q_{x}, q_{z}$ son las cargas de los quarks que componen los protones. Por lo tanto, una imagen global del espaciotiempo, sujeta a la simetría de rotación, significa que la dimensión compacta $(\mathrm{T})$ tiene el carácter de un radio o un intervalo de tiempo y, por tanto, actúa como un escalar, pero puede tener tres espacios internos -como grados de libertad. Las condiciones: la suma de las cargas de los quarks y la de suma de sus cuadrados significa que deben satisfacer una simetría de rotación SO (3). Los protones, por tanto, compuestos de quarks, son, pues, fundamentales en la TTS para nuestro universo diestro. 
Si uno se concentra por un momento en la acción de Hilbert-Einstein, sobre la expansión de la EQ de Lagrange y de la selección de aquellas partes que desaparecen en el marco de la caída libre en el modelo de TTS, obtenemos (en unidades ESU).

$$
\begin{aligned}
(16 \pi G)^{-1} K_{\text {field }} & =\frac{R}{16 \pi G}+\left.\frac{\boldsymbol{E}^{2}-\boldsymbol{B}^{2}}{8 \pi}\right|_{E=i B} \\
= & -\frac{g^{2}}{2 \pi G}+\frac{S_{E=i B}^{2}}{u_{0} c^{2}}
\end{aligned}
$$

donde $\boldsymbol{S}=0$, se asocia a los campos de la gravedad. Esta expresión cumple el principio de equivalencia, porque ambos términos desaparecen en un sistema de caída libre.

\section{APÉNDICE: ELECTRODINÁMICA QUIRAL}

Comúnmente, el electromagnetismo de Maxwell es tratado en una aproximación lineal que en muchos casos es suficiente para explicar los resultados de los experimentos. La premisa que en este trabajo de unificación se plantea es que una onda electromagnética propagándose a través de un medio es influenciada por este último y viceversa.

La idea anterior se parece al principio de Mach. Se le recuerda al lector que dicho principio sugiere que la masa de un cuerpo es debida a la influencia de todas las estrellas del universo en ese cuerpo. Expresada en términos matemáticos, esta dependencia toma la forma $m(x)=\xi[1 / T(x)]$ donde $\mathrm{m}(\mathrm{x})$ es la masa de partícula, $\xi$ una constante de acoplamiento y $1 / T$ es la masa generada por el campo a través de la quiralidad. La idea central entonces es no considerar la masa de una partícula como una cantidad fija e intrínseca, sino más bien pensarla como una cantidad variable dependiente del campo en el cual se mueve.

En este apéndice se construye la electrodinámica en espacio curvo. Para ello se parte con las ecuaciones de Maxwell en un Sistema Arbitrario de Coordenadas.

$$
\begin{gathered}
\partial_{\gamma} F_{\alpha \beta}+\partial_{\alpha} F_{\beta \gamma}+\partial_{\beta} F_{\gamma \alpha}=0 \\
\frac{1}{\sqrt{|g|}} \partial_{\beta}\left((\sqrt{|g|}) F^{\alpha \beta}\right)=J^{\alpha}
\end{gathered}
$$

Donde $F_{\alpha \beta}$ es el tensor de campo electromagnético y $j^{\alpha}$ es la densidad de corriente en 4 dimensiones. Claramente, en un sistema inercial local las ecuaciones anteriores se reducen a las ecuaciones estándares en el vacío. Para simplificarlas, se introduce un sistema de coordenadas ortogonal-temporal en el cual: $g_{\lambda 4}=0$.

Se introducen los tensores antisimétricos $H^{\alpha \beta}$ y $B^{\alpha \beta}$ tal que $F^{\alpha \beta}=H^{\alpha \beta} / \sqrt{-g_{44}}=B^{\alpha \beta}$ y los vectores $D^{\alpha}$ y $E^{\alpha}$ por $F^{\alpha 4}=-D^{\alpha} / \sqrt{-g_{44}}=E^{\alpha} / g_{44}$.

Las expresiones covariantes correspondientes son:

$$
F_{\alpha \beta}=g_{\alpha \gamma} g_{\beta \delta} F^{\gamma \delta}=g_{\alpha \gamma} g_{\beta \delta} B^{\gamma \delta}=\frac{H^{\alpha \beta}}{\sqrt{-g_{44}}}=B_{\alpha \beta}
$$

$$
F_{\alpha 4}=g_{\alpha \gamma} g_{4 \delta} F^{\gamma \delta}=g_{\alpha \beta} \sqrt{-g_{44}} D^{\beta}=\sqrt{-g_{44}} D_{\alpha}=E_{\alpha}
$$

Esto permite escribir las ecuaciones de Maxwell en la forma

$$
\begin{gathered}
\partial_{\gamma} B_{\alpha \beta}+\partial_{\alpha} B_{\beta \gamma}+\partial_{\beta} B_{\gamma \alpha}=0 \\
\frac{1}{\sqrt{-g}} \partial_{\beta}\left(\sqrt{-g} H^{\alpha \beta}\right)-\frac{1}{\sqrt{-g}} \partial_{t}\left(\sqrt{-g} D^{\alpha}\right)=\rho u^{\alpha} \\
\frac{1}{\sqrt{-g}} \partial_{\alpha}\left(\sqrt{-g} D^{\alpha}\right)=\rho
\end{gathered}
$$

Una manera de escribirlas en una forma más familiar, se introducen los vectores duales correspondientes a los tensores respectivos por la prescripción estándar, dando como resultado

$$
\begin{aligned}
& B^{1}=\frac{1}{\sqrt{-g}} B_{23}, B^{2}=\frac{1}{\sqrt{-g}} B_{3} 1, B^{3}=\frac{1}{\sqrt{-g}} B_{12} \\
& H_{1}=\frac{1}{\sqrt{-g}} H^{23}, H_{2}=\frac{1}{\sqrt{-g}} H^{31}, H_{3}=\frac{1}{\sqrt{-g}} H^{12}
\end{aligned}
$$

Finalmente haciendo las sustituciones respectivas se tienen las ecuaciones vectoriales de Maxwell sin cargas

$$
\nabla \times \boldsymbol{E}=-\frac{1}{\sqrt{-g}} \partial_{t}(\sqrt{-g} \boldsymbol{B}), \nabla \cdot \boldsymbol{B}=0
$$




$$
\nabla \times \boldsymbol{H}=\frac{1}{\sqrt{-g}} \partial_{t}(\sqrt{-g} \boldsymbol{D}), \nabla \cdot \boldsymbol{D}=\rho
$$

La conexión entre las ondas electromagnéticas en un medio y las mismas ondas en el vacío pero en un espacio curvo emerge claramente. Si g no depende del tiempo y si se tiene $\mu=\varepsilon=-\left(-\mathrm{g}_{44}\right)^{-1}$, entonces el campo, responsable por la curvatura del espacio, se comporta como un medio con la permitividad dieléctrica y susceptibilidad magnética dada por la ecuación anterior. La conjetura que se plantea en esta investigación es que $\varepsilon,{ }_{0}$ y $\mu_{0}$ en el espacio curvado son definidos como los operadores: $\varepsilon_{0}(1+T \nabla \times)$ y $\mu_{0}(1+T \nabla \times)$ donde $\mathrm{T}$ es el factor quiral que permite la torsión del campo dando lugar a la creación de las partículas.

\section{Las ecuaciones de campo}

En un sistema de referencia, donde el medio está en reposo, las relaciones constitutivas quirales de BornFederov son:

$$
\begin{gathered}
\boldsymbol{D}^{(0)}=\varepsilon \boldsymbol{E}^{(0)}+\varepsilon T \nabla \times \boldsymbol{E}^{(0)} \\
\boldsymbol{B}^{(0)}=\mu \boldsymbol{H}^{(0)}+\mu T \nabla \times \boldsymbol{H}^{(0)}
\end{gathered}
$$

en el sistema en reposo $S^{o}$ del medio. Se restringe el estudio a medios homogéneos y no dispersivos donde $\varepsilon$, $\mu$ y $T$ son constantes. Las ecuaciones de Maxwell en este marco, en ausencia de cargas son

$$
\begin{array}{cl}
\nabla \cdot \boldsymbol{D}^{(0)}=0 & \nabla \times \boldsymbol{H}^{(0)}=\boldsymbol{D}_{, 0}^{(0)} \\
\nabla \cdot \boldsymbol{B}^{(0)}=0 & \nabla \times \boldsymbol{E}^{(0)}=-\boldsymbol{B}_{, 0}^{(0)}
\end{array}
$$

donde $a_{, 0}=\frac{\partial a}{\partial t}$. Las ecuaciones de Maxwell pueden ser escritas en términos de los campos $\boldsymbol{E}^{(0)}$ y $\boldsymbol{B}^{(0)}$

$$
\begin{gathered}
\nabla \cdot \boldsymbol{E}^{(0)}=0, \nabla \times \boldsymbol{B}^{(0)}=\mu \boldsymbol{J}^{(0)}+\mu \varepsilon \boldsymbol{E}_{, 0}^{(0)} \\
\nabla \cdot \boldsymbol{B}^{(0)}=0, \nabla x E^{(0)}=-\boldsymbol{B}_{, 0}^{(0)}
\end{gathered}
$$

donde $\boldsymbol{J}^{(0)}=\varepsilon T \nabla \times \boldsymbol{K}^{(0)}$ con

$$
\boldsymbol{K}^{(0)}=2 \boldsymbol{E}_{, 0}^{(0)}+T \nabla \times \boldsymbol{E}_{, 0}^{(0)}=2 \boldsymbol{E}_{, 0}^{(0)}-T \boldsymbol{B}_{, 00}^{(0)}
$$

También se tiene

$$
\boldsymbol{J}^{(0)}=\varepsilon T\left(-2 \boldsymbol{B}_{, 00}^{(0)}-T \nabla^{2} E_{, 0}^{(0)}\right)
$$

Las ecuaciones de Maxwell rescritas anteriormente son formalmente similares a las ecuaciones de Maxwell en un medio normal con densidad de corriente $\boldsymbol{J}^{(0)} \mathrm{y}$ densidad de carga $\rho^{(0)}=0$ que obedece la ecuación de continuidad de la carga $\nabla \cdot \boldsymbol{J}^{(0)}+\rho_{, 0}{ }^{(0)}=0$. Se llamará $\boldsymbol{J}^{(0)}$ a la corriente quiral en el marco de referencia en reposo. En un sistema arbitrario $S$, las ecuaciones de Maxwell pueden ser escritas $\frac{\partial a}{\partial x^{\alpha}}=a_{, \alpha},[41]$.

$$
\begin{gathered}
F_{, \alpha}^{\alpha \lambda}-(\mu \varepsilon-1) F_{, \gamma}^{\lambda \alpha} u_{\alpha} u^{\gamma}=\mu J^{\lambda} \\
F_{\alpha \beta, \gamma}+F_{\gamma \alpha, \beta}+F_{\beta \gamma, \alpha}=0
\end{gathered}
$$

respectivamente. Aquí $u_{\alpha}$ es la velocidad uniforme del medio, $F_{\alpha \beta}$ es el tensor del campo electromagnético con componentes $F_{0 i}=E_{i} \quad F_{i j}=-\varepsilon_{i j k} B_{k}$. También se introduce el dual del tensor de campo

$$
G^{\alpha \beta}=\frac{1}{2} \varepsilon^{\alpha \beta \gamma \delta} F_{\gamma \delta}
$$

con componentes $G_{0 i}=B_{i} \quad G_{i j}=+\varepsilon_{i j k} E_{k}$, de modo que la corriente quiral puede ser escrita como

$$
J^{\lambda}=\varepsilon T \varepsilon^{\alpha \lambda \rho \sigma} u_{\alpha} K_{\rho, \sigma}
$$

$\operatorname{con} \varepsilon^{0123}=+1$ 甲 $K^{\rho}=2 F^{\rho}-T G^{\rho}$.

Aquí usamos la definición: $F^{\rho}=F^{\rho \alpha} u_{\alpha} ; G^{\rho}=G^{\rho \alpha} u_{\alpha}$ y donde la operación punto (.) es definida por $a=u^{\alpha} a_{, \alpha}$, la cual se reduce a la derivada temporal ordinaria en el medio en reposo. En este marco $F^{i}$ se reduce a las componentes del campo eléctrico (y $F^{0}$ desaparece) y $G^{i}$ a las del campo magnético (y $G^{o}$ desaparece).

La corriente quiral se puede escribir en la forma

$$
J^{\alpha}=\varepsilon T\left(-2 G^{\alpha}+T h^{\mu v} F_{, \mu v}^{\alpha}\right)
$$

donde $h^{\mu v}$ está relacionado al tensor métrico $g^{\mu v}$ por $h^{\mu v}=g^{\mu v}-u^{\mu} u^{v}$

Se hace notar que la ecuación de continuidad es considerada. $\mathrm{J}^{\lambda}, \lambda=0$. Efectuando una contracción con $\mathrm{u}^{\lambda} \mathrm{y}$ notando que 
$u^{\lambda} \mathrm{J}^{\lambda}=0$ se tiene $F^{\alpha},{ }_{\alpha}=F^{\alpha \lambda}, u_{\lambda}=0$. Este resultado no es nada más que $\nabla \cdot \boldsymbol{E}^{(0)}=0, \nabla \times \boldsymbol{B}^{(0)}=\mu \boldsymbol{J}^{(0)}+\varepsilon \mu \boldsymbol{E}_{, 0}{ }^{(0)}$ en el medio en reposo.

El inverso de $G_{\beta \delta}$ es

$$
F^{\alpha \lambda}=-\frac{1}{2} \varepsilon^{\alpha \lambda \beta \delta} G_{\beta \delta}
$$

Si se introduce esta relación en $F^{\alpha \lambda}, \alpha-(\varepsilon \mu-1) F^{\lambda \alpha}$, ${ }_{\gamma} u_{\alpha} u^{\gamma}=\mu J^{\lambda}$ y haciendo la contracción con $\varepsilon_{\rho \lambda \sigma \gamma}$ se obtiene

$$
G_{\sigma \gamma, \rho}+G_{\rho \sigma, \gamma}+G_{\gamma p, \sigma}-(\mu \varepsilon-1) \varepsilon_{\rho \lambda \sigma \gamma} \dot{F}^{\lambda}=\mu \varepsilon_{\rho \lambda \sigma \gamma} J^{\lambda}
$$

donde se ha usado la identidad

$$
\varepsilon^{\lambda \alpha \beta \mu} \varepsilon_{\lambda \rho \sigma \gamma}=-\delta_{\rho}^{\alpha} \delta_{[\sigma}^{\beta} \delta_{\gamma]}^{\mu}+\delta_{\sigma}^{\alpha} \delta_{[\rho}^{\beta} \delta_{\gamma]}^{\mu}-\delta_{\gamma}^{\alpha} \delta_{[\rho}^{\beta} \delta_{\sigma]}^{\mu}
$$

con $[\sigma \gamma]=\sigma \gamma-\gamma \sigma$. La ecuación homogénea $F_{\alpha \beta, \gamma}+F_{\gamma \alpha, \beta}+F_{\beta \gamma, \alpha}=0$ se transforma en $G_{, \gamma}^{\gamma \sigma}=0$ que inmediatamente sigue de la contracción de la ecuación homogénea con $\varepsilon^{\lambda \sigma \alpha \beta}$. Para obtener la ecuación de onda del tensor de campo se diferencia $F_{\alpha \beta, \gamma}+F_{\gamma \alpha, \beta}+F_{\beta \gamma, \alpha}=0$ con respecto a $\gamma$, y se usa $F_{, \alpha}^{\alpha \lambda}-(\varepsilon \mu-1) F_{, \gamma}^{\lambda \alpha} u_{\alpha} u^{\gamma}=\mu J^{\lambda}$ para obtener

$$
F_{\alpha \beta, \gamma}^{\gamma}-(\mu \varepsilon-1) \ddot{F}_{\alpha \beta}=-\mu\left(J_{\alpha, \beta}-J_{\beta, \alpha}\right)
$$

en este resultado final se utiliza la relación $F_{\alpha \beta}=u^{\gamma} F_{\alpha \beta, \gamma}=F_{\alpha, \beta}-F_{\beta, \alpha}$. La ecuación de onda $F_{\alpha \beta, \gamma}{ }^{\gamma}+(\varepsilon \mu-1) F_{\alpha \beta}=-\mu\left(J_{\alpha, \beta}-J_{\beta, \alpha}\right)$ es el resultado fundamental de este trabajo de investigación que permite la unificación del electromagnetismo con la gravitación. Esta ecuación de onda de segundo orden en tiempo y espacio permite obtener la propagación de gravitones si $\left(J_{\alpha, \beta}-J_{\beta, \alpha}\right)=0$ y de fotones si $T \equiv 0$ respectivamente. Para examinar el caso de gravitones con spin 2 el primer miembro de la ecuación de onda (16) es

$$
F_{\alpha \beta, \gamma}^{\gamma}+(\varepsilon \mu-1) F_{\alpha \beta}=0
$$

que corresponde al caso de corriente quiral igual a cero, esto es o sea

$$
J^{\alpha}=\varepsilon T\left(-2 G^{\alpha}+\ddot{T} h^{\mu \nu} \dot{F}_{, \mu \nu}^{\alpha}\right)=0
$$

$$
\varepsilon T 2 G^{\alpha}=\ddot{*} T h^{\mu v} F_{, \mu v}^{\alpha}
$$

En un marco de referencia en reposo $S^{o}$, esta ecuación se simplifica a la ecuación de Beltrami si hacemos $T \equiv \frac{\hbar}{m c}$,

$$
\nabla \times \boldsymbol{E}^{(0)}+\frac{2}{T} \boldsymbol{E}^{(0)}=0
$$

es trivial obtener de la ecuación de onda (17) con $\partial / \partial t=i \omega$, y la velocidad de la luz dada por $c=(\varepsilon \mu)^{-1 / 2}$ la expresión $k_{0}=\frac{\omega}{c}$ tal que $m c T=2 \hbar$. Esto corresponde a partículas con spin 2. Se observa además que de la ecuación $\nabla \times \boldsymbol{E}^{(0)}=-\boldsymbol{B}_{, 0}^{(0)}=-i \omega \boldsymbol{B}^{(0)} 1$ se tiene entonces que $\boldsymbol{E}^{(0)}=i \omega \boldsymbol{B}^{(0)}$, o sea los campos son paralelos en el espacio tridimensional con el vector de Poynting $\boldsymbol{E}^{(0)} \times \boldsymbol{B}^{(0)}=0$. Esto implica una gran dificultad en detectar este tipo de partículas con detectores usuales de radiación.

El caso de ondas electromagnéticas normales es analizado con la ecuación

$$
\nabla \times \nabla \times\left(1-k_{0}^{2} T^{2}\right) \boldsymbol{E}-2 k_{0}^{2} T \nabla \times \boldsymbol{E}-k_{0}^{2} \boldsymbol{E}=0
$$

$\mathrm{Si} \mathrm{T}=0$ se obtiene la usual ecuación de onda en un medio normal y homogéneo que se encuentra en los textos de electromagnetismo, $\nabla \times \nabla \times \boldsymbol{E}-k_{0}^{2} \boldsymbol{E}=0$, ondas que al ser tratadas como partículas se tiene que el spin es igual a uno.

Si $k_{0} T \leq 1$ se obtienen las ondas quirales circularmente polarizadas que se propagan en medios electromagnéticos complejos y en medios biológicos (por ejemplo, ondas en el tejido cerebral debido a las microondas de teléfonos celulares).

Si $k_{0} T \geq 1$ o mucho mayor que uno, se obtiene una ecuación de tipo Beltrami que entre otras situaciones físicas puede modelar las ondas en una estrella de neutrones, explicar las llamaradas solares donde la corriente es paralela al campo magnético. Esta relación también permite obtener el radio del universo si $T=\hbar / m c$ y $\mu$ es la masa del fotón en el espacio curvado de Einstein. 
Usando un modelo de fluido de fotones, permite obtener $\operatorname{rot} \boldsymbol{B}=\lambda B$ que puede calcular la distancia del Sol a cada uno de los planetas del Sistema Solar.

Además puede explicar: la generación de bolas de luz, la formación de galaxias en espiral y las ondas EM quirales como autoestados de moléculas de ADN.

Se puede demostrar rigurosamente que con ondas electromagnéticas donde $\boldsymbol{E}$ es perpendicular a $\boldsymbol{B}$, $(\boldsymbol{E} \perp \boldsymbol{B})$ la ecuación tensorial de Einstein, con el tensor de Maxwell $T_{\mu \nu}$ es

$$
R_{\mu v}-\frac{1}{2} g_{\mu \nu} R=-\kappa T_{\mu \nu}
$$

Es transparente al campo de manera que estos modos de propagación no permiten la unificación ya que al hacer lo hecho por primera vez por Einstein para el caso usual de campos de Maxwell $(\boldsymbol{E} \perp \boldsymbol{B})$, o sea $(\mathbf{E} \cdot \mathbf{B}=0)$

$$
\bar{g}_{\mu v}=g_{\mu v}+F_{\mu v}
$$

Ya el determinante de $F_{\mu \nu}$ es igual a cero $\left(\operatorname{det} F_{\mu \nu}=2(\boldsymbol{E} \cdot \boldsymbol{B})^{2}=0\right)$ se tiene que

$$
d s^{2}=\bar{g}_{\mu v} d x_{\mu} d x_{v}=\bar{g}_{\mu v} d x_{\mu} d x_{v}
$$

Esta es la razón del porqué Einstein no pudo obtener la anhelada unificación del electromagnetismo y la gravitación.

Esta formulación es un enlace entre la Teoría Cuántica y la Relatividad General, siendo clave el concepto de campo Beltrami como fundamental en la creación de partículas. En la Física actual, ambas teorías están profundamente cimentadas en marcos espacio-tiempo distintos. La primera en el espacio-tiempo de Minkowski, y la segunda en el espacio-tiempo curvado.

Tal como A. Wheeler lo hizo notar, los intentos de unificación y el desafío han sido la introducción de la mecánica cuántica con spin $1 / 2$ en la Relatividad General, por un lado, y la introducción de la curvatura en Mecánica Cuántica por otro. La ecuación de onda $F_{\alpha \beta, \gamma}^{\gamma}(T)+(\varepsilon \mu-1) \stackrel{F_{\alpha \beta}}{ }(T)=-\mu\left(J_{\alpha, \beta}(T)-J_{\beta, \alpha}(T)\right)$ que es la generalización de la ecuación de Klein-Gordon permite esta conexión, si la transformamos poniendo en evidencia el factor de spin $1 / 2$.
La ecuación tensorial de Maxwell (12) $F_{, \alpha}^{\alpha \lambda}-(\mu \varepsilon-1) F_{, \gamma}^{\lambda \alpha} u_{\alpha} u^{\gamma}=\mu J^{\lambda}$, se puede transformar a

$$
O\left(F_{, \alpha}^{\alpha \lambda}-(\mu \varepsilon-1) F_{, \gamma}^{\lambda \alpha} u_{\alpha} u^{\gamma}\right)=\mu J_{e p}^{\lambda}
$$

donde el tensor corriente, $J_{e p}^{\lambda}$, corresponde a la corriente quiral del electrón $(\mathrm{T}>0)$ o positrón $(\mathrm{T}<0)$

$$
J_{e p}^{\lambda}=\varepsilon\left([1-O] \dot{F}^{\lambda}-2 T G^{\lambda}\right)
$$

donde

$$
O \equiv 1+T^{2} u^{\alpha}\left(u_{, \alpha}^{\alpha}\right)_{, \alpha}
$$

manipulando las ecuaciones (22), (23) y (24), se puede obtener la ecuación de onda

$$
\begin{aligned}
& O F_{\alpha \beta, \gamma}^{\gamma}(T)+O(\varepsilon \mu-1) F_{\alpha \beta}(T)= \\
& =-\mu\left(J_{(e p) \alpha, \beta}(T)-J_{(e p) \beta, \alpha}(T)\right)
\end{aligned}
$$

Explícitamente la ecuación de onda puede ser linealizada siguiendo la genial línea de raciocinio de P. Dirac (que a partir de la ecuación de segundo orden de Klein Gordon desarrolla las matrices $\alpha, \beta$ para obtener la ecuación de primer orden de Dirac, donde el spin $1 / 2$ aparece en forma precisa).

Siguiendo el mismo raciocinio de Dirac, hacemos $O=0$, y al integrar una vez la ecuación (25) se tiene

$$
\dot{F}^{\lambda}=2 T G^{\lambda}
$$

que en el sistema de referencia del electrón o positrón corresponde a

$$
\nabla \times \boldsymbol{E}^{(0)}+\frac{1}{2 T} \boldsymbol{E}^{(0)}=0
$$

donde se ha hecho $k_{0} T= \pm 1$. Aquí se considera el principio de incertidumbre de Heisenberg $p T= \pm \hbar / 2=m c T$. Se hace notar que este resultado general se obtiene también de la ecuación (19) que permite obtener relaciones isomórficas que conducen a una manera inédita de obtener la ecuación de Dirac para una partícula elemental como el electrón o positrón a partir de la obtención de la ecuación de Beltrami (free force). La onda inicialmente con spin 1 y energía $\hbar \omega$ es transformada en campos Beltrami de spin $+1 / 2$ y $-1 / 2$. En otras palabras, si el sistema de ecuaciones de Maxwell es multiplicado por la matriz de Pauli permite obtener en forma rigurosa y original 
la Ecuación de Dirac. Esta deducción se muestra en el artículo correspondiente.

Usando álgebra cuaterniónica en un sistema de referencia fijo a la partícula (onda de luz curvada sobre sí misma), de la ecuación anterior se obtiene sin aproximaciones la ecuación de Dirac. En ella aparece en forma explícita el spin de la partícula, cuando $k_{0} T=1$. O sea si $T=\hbar / 2 m c$ entonces

$$
k_{0} T=\omega T / c=1=\hbar \omega / 2 m c^{2} \Rightarrow E=2 m c^{2} .
$$

De esta forma, a partir del escalar T, se obtiene la ecuación fundamental de Einstein entre materia y energía. El escalar $\mathrm{T}$ se encuentra implícito en

$$
F_{\alpha \beta, \gamma}^{\gamma}(T)+(\varepsilon \mu-1) \ddot{F}_{\alpha \beta}(T)=-\mu\left(J_{\alpha, \beta}(T)-J_{\beta, \alpha}(T)\right)
$$

$\mathrm{Si} \mathrm{T}=0$ se tiene el espacio plano de cuatro dimensiones de Minkowski, base del electromagnetismo de Maxwell y de la Relatividad Especial.

Recientes modelos que incluyen supercuerdas cuánticas son caminos alternativos que consideran partículas extendidas, es decir, no puntuales que conducen a operadores espaciotiempo no diferenciables y a geometría no conmutativa, pero la teoría propuesta aquí es más económica, más simple en dimensiones y en la potente idea de que los campos electromagnéticos con $\boldsymbol{E} \| \boldsymbol{B}$ son la verdadera fuente para la gravitación

\section{CONCLUSIONES}

Como una alternativa a la teoría cuántica de la gravedad, aún no descubierta, la TTS ha sido propuesta, que postula unificar la gravedad con el EM teniendo como corolario fundamental la ecuación cuántica de Dirac.

En este programa de unificación en el cual el electromagnetismo quiral juega el rol central, la TTS ha sido derivada de las ecuaciones originales de Einstein -Hilbert $G_{\mu v}=\kappa T_{\mu \nu}$, donde el tensor de Einstein no ha sido modificado. El tensor EM en cambio es quiral y la masa de las partículas es de naturaleza electromagnética. Para el caso del electrón se tiene como consecuencia que por primera vez se obtiene la ecuación de Dirac a partir de ondas EM con el campo eléctrico paralelo espacialmente al campo magnético. Como modelo del universo se propuso una interfaz o membrana de separación entre dos regiones enantioméricas de un universo cerrado, o un universo derecho y uno universo izquierdo, relacionados por un elemento de simetría PCT (paridad, carga, tiempo) a lo largo de la interfaz. Las ecuaciones de EinsteinHilbert fueron estudiadas bajo el enfoque quiral y se han analizado la electrodinámica quiral y la gravedad en la era de Planck.

\section{AGRADECIMIENTOS}

Se agradecen las fructíferas discusiones sobre el tema con los colegas del Instituto de Alta Investigación y de la Escuela Universitaria de Ingeniería Eléctrica - Electrónica de la Universidad de Tarapacá, Arica, Chile.

\section{REFERENCIAS}

[1] H. Torres-Silva. "Chiroplasma surface wave". Electromagnetic of Chiral Bi-Isotropic and BiAnisotropic Media. CHIRAL '96. Proceeding of Nato Series, Moscú. 1996.

[2] H. Torres-Silva, P. Sakanaka and N. Reggiani. "The Effect of Chirality on a Plasma Media". Rev. Mex. de Física. Vol. 42, pp. 989-1000. 1996.

[3] H. Torres-Silva and C. Villarroel González. "Electromagnetic properties of a Chiral-Plasma Medium". Pramana-Journal of Physics. Vol. 49, pp. 431-442. 1997.

[4] H. Torres-Silva. "Chiroplasma surface wave". A. Priou, editor: Advances in Complex Electromagnetic. Materials. Vol. 28, pp. 249-258. Kluwer Academic Publishers. 1997.

[5] H. Torres-Silva. "Electromagnetic Waves in a Chiral Plasma". Journal of the Physical Soc. of Japan. Vol. 67, pp. 850-857. 1998.

[6] H. Torres-Silva. "Propagación de ondas pulsadas en un chiroplasma magnetizado". Rev. Mex. de Física. Vol. 44, pp. 53-58. 1998.

[7] H. Torres-Silva. "Convective Instabilities of Transverse Wave In Magnetized Chiral Media". J. Plasma Res. Vol. 1, p. 395. 1999.

[8] H. Torres-Silva y M. Zamorano Lucero. "Ecuación de Onda de Schrödinger para una fibra Óptica Chiral”. Revista Mexicana de Física Vol. 46, pp. 62-66. 2000. 
[9] A. Assis and H. Torres-Silva. "Relation between Maxwell Equations and Weber Force". Pranama Journal of Physics. Vol. $54 \mathrm{~N}^{\circ}$ 6, pp. 1-12. 2000.

[10] H. Torres-Silva and M. Zamorano Lucero. "Polarized spatial solitons in cubic chiral materials". PIER 2002. Progress In Electromagnetics Research. Cambridge, Massachusetts, USA. July 1-5. 2002.

[11] C. Villarroel González y H. Torres-Silva. "Difracción en el borde de un semiplano inmerso en un medio quiral bianisotrópico". Rev. Mex. de Física. Vol. 47, pp. 136-141. 2001.

[12] H. Torres-Silva and M. Zamorano Lucero. "Chiral effects on optical solitons". Mathematics and Computers in Simulations. Vol. 62, pp. 149-161. 2003.

[13] M. Zamorano Lucero y H. Torres-Silva. "Efecto de la quiralidad sobre solitones polarizados en un medio anisotrópico". Revista Mexicana de Física. Vol. $49 \mathrm{~N}^{\mathrm{o}}$ 1, pp. 20-27. 2003.

[14] H. Torres-Silva and M. Zamorano Lucero. "Nonlinear polarization and chiral effects in birefringent solitons". Pramana Journal of Physics. Vol. 62 $\mathrm{N}^{\circ}$ 1, p. 37. 2004.

[15] M. Zamorano Lucero y H. Torres-Silva. "Sar inducido en un modelo bioplasmático quiral por radiación de teléfonos celulares". Revista Mexicana

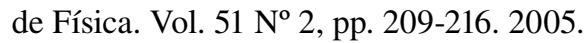

[16] H. Torres-Silva. "FDTD chiral brain tissue model for specific absorption rate determination under radiation from mobile phones at 900 and 1800 MHz". Phy in Med. and Biol. Vol. 51, pp. 16611672. 2006.

[17] L.A. Ambrosio, H.E. Hernández and H. Torres-Silva. "Guided modes in metamaterial slabs". Ingeniare. Rev. chil. ing. Vol. 14 N³ 3, pp. 291-298. 2006.

[18] H. Torres-Silva, C. Villarroel González and F. Jiménez-Muñoz. "Electromagnetic waves at the plane boundary between two chiral media". Ingeniare. Rev. chil. ing. Vol. 15 N$^{\mathrm{o}}$ 1, pp. 101-110. 2007.

[19] T. Appelquist. Modern Kaluza-Klein Theories. Frontier in Physics. Addisson-Wesley. 1987.
[20] C.W. Misner, K.S. Thorne and J.A. Wheeler. Gravitation, Ch. 13, Freeman, pp. 310-311. 1973.

[21] R. Wald, General Relativity. Cap. 14. Ed. University Chicago Press. 1984.

[22] H. Torres-Silva. "A new relativistic field theory of the electron". Ingeniare. Rev. chil. ing. Vol. 16 $\mathrm{N}^{\mathrm{o}}$ 1, pp. 111-118. 2008.

[23] H. Torres-Silva. "Spin and relativity: a semiclassical model for electron spin”. Ingeniare. Rev. chil. ing. Vol. $16 \mathrm{~N}^{\mathrm{o}}$ 1, pp. 72-77. 2008.

[24] H. Torres-Silva. "A metric for a chiral potential field”. Ingeniare. Rev. chil. ing. Vol. $16 \mathrm{~N}^{\mathrm{o}} 1$, pp. 91-98. 2008.

[25] H. Torres-Silva. "Maxwell's theory with chiral currents". Ingeniare. Rev. chil. ing. Vol. $16 \mathrm{~N}^{\circ} 1$, pp. 31-35. 2008.

[26] H. Torres-Silva. "The close relation between the Maxwell system and the Dirac equation when the electric field is parallel to the magnetic field". Ingeniare. Rev. chil. ing. Vol. $16 \mathrm{~N}^{\circ} 1$, pp. 43-47. 2008.

[27] J. Earman and C. Glymour. "Einstein and Hilbert: Two Months in the History of General Relativity". Archive for History of Exact Sciences. Vol. 19, p. 291. 1978.

[28] L. Corry, J. Renn and J. Stachel. "Belated Decision in the Hilbert-Einstein Priority Dispute”. Science. Vol. 278, p. 1270. 1997.

[29] J. Renn and J. Stachel. Hilbert's Foundation of Physics: From a Theory of Everything to a Constituent of General Relativity. Preprint of Max-Planck-Institut für Wissenschaftsgeschichte. No 118. 1999.

[30] V.P. Vizgin. "On the discovery of the gravitational field equations by Einstein and Hilbert: new materials". Physics-Uspekhi. Vol. 44 N 12 , p. 1283. 2001.

[31] A. Einstein. "The Collected Papers of Albert Einstein”. Eds. R. Schulmann. Princeton, N.Y. Princeton Univ. Press. No 8. 1998.

[32] T. Sauer. "The Relativity of Discovery: Hilbert's First Note on the Foundations of Physics". Archive 
for History of Exact Sciences. Vol. 53, pp. 529-575. 1999.

[33] A. Einstein. "Do Gravitational Fields Play an Essential Part in the Structure of the Elementary Particles of Matter? The Principle of Relativity". Dover, pp. 191-198. 1952.

[34] H. Torres-Silva. "Extended Einstein's theory of waves in the presence of space-time tensions". Ingeniare. Rev. chil. ing. Vol. $16 \mathrm{~N}^{\mathrm{o}} 1$, pp. 78-84. 2008.

[35] H. Torres-Silva. "Einstein equations for tetrad fields". Ingeniare. Rev. chil. ing. Vol. $16 \mathrm{~N}^{\circ} 1$, pp. 85-90. 2008.

[36] H. Torres-Silva. "Chiral universes and quantum effects produced by electromagnetic fields". Ingeniare. Rev. chil. ing. Vol. $16 \mathrm{~N}^{\circ} 1$, pp. 99-110. 2008.
[37] J.E. Brandenburg. "A model cosmology based on gravity - electromagnetism unification, Astrophys". Space Sci. Vol. 227 No 1/2, pp. 133144. 1995.

[38] H.E. Puthoff. "Gravity as a zero-point fluctuation force". Phys. Rev. A. Gen. Phys. Vol. 39 No 5, pp. 2333-2342. 1989.

[39] A.D. Sakharov. Vacuum quantum fluctuations in curved space and the theory of gravitation. Sov. Phys. Dokl. Vol. 12 No 2, pp. 1040-1041. 1967.

[40] Y. B. Zel'dovich. Cosmological constant and elementary particles. Sov. Phys. JETP Lett. Vol. 6, pp. 316-317. 1967.

[41] S. Ragusa. "First-order conservation Laxs in Chiral medium". Brazilian Journal of Phycs. Vol. 26, pp. 411-418. 1996. 